\title{
RE-ASSESSMENT AND UPDATE OF BULK LONGSHORE SEDIMENT TRANSPORT FORMULAS
}

\author{
João Mil-Homens ${ }^{1}$, Roshanka Ranasinghe ${ }^{1,2,3}$, Jaap van Thiel de Vries ${ }^{1,4}$ and Marcel Stive

\begin{abstract}
Longshore sediment transport (LST) is one of the main drivers of beach morphology. Bulk LST formulas are routinely used in coastal management/engineering studies to assess LST rates and gradients. Over 50 years of research has resulted in several bulk LST formulas that have been tested with varying levels of rigor. In this study, the predictive skill of one of the most recent bulk LST formulas (Bayram et al., 2007) is evaluated. The calibration coefficients in the formula are improved using a least-squares optimization algorithm, resulting in a significant improvement in predictive skill. The generality of the improved formula is verified via the statistical methods of bootstrapping and cross-validation.
\end{abstract}

Keywords: Longshore sediment transport; LST bulk formulas; LST data set

\section{INTRODUCTION}

In the surfzone, breaking waves generate several horizontal and vertical current patterns that can stir up and transport sediment. Sometimes this transport results only in local sand redistribution. In other occasions there is extensive longshore sediment displacement, moving enormous quantities of sand along the coast. This longshore sediment transport (LST) is one of the most important processes that control coastal morphology, and determines to a large extent whether shores erode, accrete or remain stable. Large and/or persistent LST rates may have other impacts, e.g., inlet closure/migration, ebb/flood delta erosion/accretion, rotation of pocket beaches, headland sand bypassing. These processes may represent a threat for populations settled in coastal regions, for man made structures built on the coastline and for the usability of waterways. The calculation of LST rates is therefore a key component on most coastal engineering/planning studies.

\section{LST models}

There are two main approaches to estimate LST:

- Bulk transport formulas - these are basic models that assume a simplified representation of the physical processes and generally use empirical coefficients for calibration. These formulas provide a quick estimate of the LST rate, with relatively few input parameters. Two of the most commonly used formulas are the CERC (CERC, 1984) and the Kamphuis (Kamphuis, 1991) formulas.

- Process based models - intend to include a large number of physical processes (shear stress, pickup, suspension, wave-current interaction, etc). Process-based models try to simulate, on a detailed way, the LST. These models often need a large number of input parameters. Examples are: the model described in Deigaard et al. (1986), UNIBEST (WL|Delft Hydraulics, 1992; Stive and Battjes, 1984) and GENESIS (Hanson, 1989).

Both approaches are useful for coastal engineers. Bulk formulations are often used to make a first guess based on limited information and process-based models are generally expected to produce more accurate estimates but require also more accurate input information and are more labor intensive.

The main goal of this study is to increase the predictive accuracy of one of the most recent LST bulk formulas, i.e., the Bayram formula (Bayram et al., 2007), by developing a new expression for the calibration coefficient. To accomplish that, an error analysis based on logarithmic values was used and the possibility of having a non-linear function as calibration coefficient was considered.

\section{DATA SET}

In this study, the data set presented in Bayram et al. (2007) was used. This data set consists on a compilation of several smaller data sets that span in time from 1953 to as recently as 2004. A large variety of methods was used in the data collection, from visual observation of wave heights, to the more sophisticated backscattering methods of measuring suspended sediment concentration. There are also measurements with different time frames, ranging from the space of a few minutes to months.

\footnotetext{
${ }^{1}$ Faculty of Civil Engineering and Geosciences, Delft University of Technology, PO Box 5048, 2600 GA Delft, The Netherlands

2 Department of Water Engineering, UNESCO-IHE, PO Box 3015, 2601 DA Delft, The Netherlands

3 Harbour, Coastal and Offshore Engineering, Deltares, PO Box 177, $2600 \mathrm{MH}$ Delft, The Netherlands

${ }^{4}$ Morphology and Sediment Dynamics, Deltares, PO Box 177, 2600 MH Delft, The Netherlands
} 
In order to be used in this study, the data sets need to include measurements of: significant wave height at the breaker, peak period, wave angle at the breaker and mean grain diameter. The data set is composed by:

- the data presented in Schoonees and Theron (1993) - the Data Set 1 referred in that study,

composed by 123 data points

- a laboratory data set with 4 points (Smith et al., 2003)

- a field data set collected in Duck, North Carolina, under high energy conditions (Miller, 1999) with 10 points

- a field data set collected in Karwar, India (Sanil Kumar et al., 2003) with 81 points

- a field data set collected in the East Coast and Gulf Coast of the USA (Wang et al., 1998) with 29 points.

An analysis of the data distribution was done. This analysis is important to access the representativeness of the data set. It also indicates areas where a limited number of data points is available.

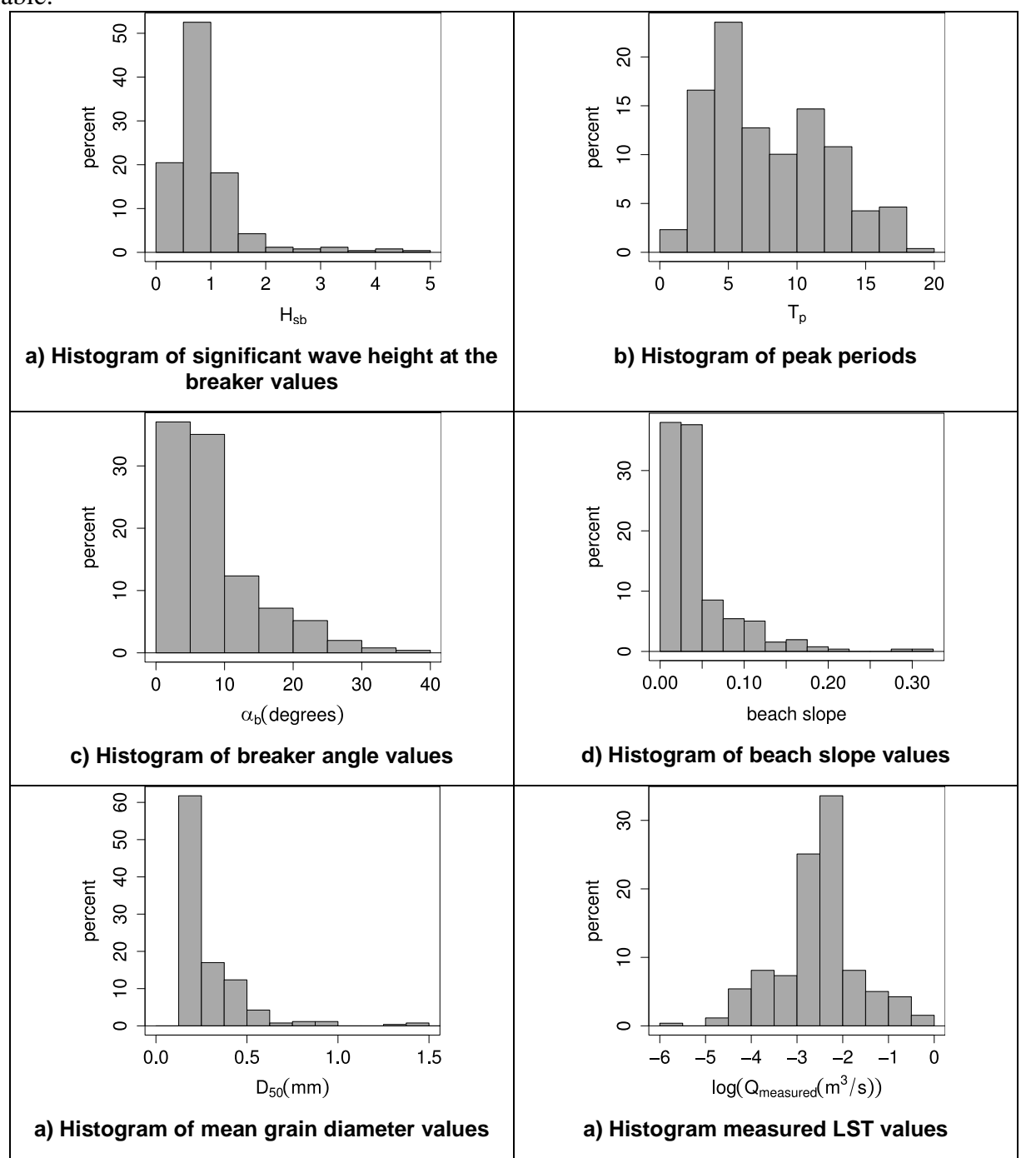

Figure 1 - Histograms of (a) significant wave height, (b) peak period, (c) breaker angle, (d) beach slope, (e) mean grain diameter and (f) measured LST

The significant wave height distribution (Figure 1a) shows that more than $70 \%$ of the data refer to waves smaller than $1 \mathrm{~m}$, and that there are very few points above $2 \mathrm{~m}$. This can be explained by the difficulty of the measurements in higher wave conditions. The peak period histogram (Figure 1b) shows a bimodal distribution, where peaks around $5 \mathrm{~s}$ and $11 \mathrm{~s}$ are observed. Regarding the wave angle at the breaker (Figure 1c) most values are less than 10 degrees. More than $70 \%$ of the data points are from 
beaches with slopes under 0.05 , which fall in the dissipative region, following Wright and Short (1984)'s classification. Approximately 52\% of the data points correspond to fine sand (D50 under $0.2 \mathrm{~mm}$ ), $42 \%$ enter the category of medium sand. LST rates (Figure 1f) are more concentrated between $10^{-3}$ and $10^{-2}$. The distribution fades gradually for smaller and higher magnitude orders.

The main shortcoming of this data set is the low number of data points in the higher transport region (e.g. between 0.1 and 10). This region is associated with events (storms) that are usually responsible for almost all significant LST during a large time period. There's also shortness of data for coarse sand and reflective beaches.

\section{THE BAYRAM FORMULA}

In the Bayram formula (Bayram et al., 2007) it is assumed that a great share of the transported sediment is in suspension (suspended load), and that the sediment becomes suspended by the action of breaking waves. After that, any type of longshore current can transport the sediment. The wave breaking stirs up the sediment and maintains an average concentration distribution $c(x, z)$ in the surf zone. In order to keep the sediment in suspension, the total work $(W)$ necessary can be given by the product of the concentration, submerged weight and fall speed $\left(w_{s}\right)$ :

$$
W=\int_{0}^{x_{b}} \int_{-h(x)}^{0} c(x, z)\left(\rho_{s}-\rho\right) g w_{s} d z d x
$$

where $x$ is a cross-shore coordinate with the origin at the shoreline and positive in the offshore direction, the subscript $b$ refers to the breaking point, $z$ is the vertical coordinate with origin at the still water level and negative underwater and $\mathrm{d}$ is the water depth. It is considered that the work $W$ is a fraction of the flux of wave energy $\left(F=E C_{g}\right)$, i.e. $W=\varepsilon F$. $(V)$ :

LST can be defined as the product of the suspended concentration and longshore current velocity

$$
Q_{l}=\int_{0}^{x_{b}} \int_{-d(x)}^{0} c(x, z) V(x, z) d z d x
$$

When a representative longshore current velocity is considered, Eq.(1), $W=\varepsilon F$, and Eq.(2) can be combined into:

$$
Q_{l}=\frac{\varepsilon}{\left(\rho_{s}-\rho\right)(1-a) g w_{s}} F \bar{V}
$$

where $V$ is the mean (or representative) longshore current velocity over the surf zone, $a$ is the porosity and $\varepsilon$ is a coefficient that represents the efficiency of the waves in keeping sand grains in suspension. In Bayram et al.(2007) the transport coefficient was estimated by performing a dimensional analysis, Bayram et al.(2007) suggested the following coefficient:

$$
\varepsilon=\left(9+4 \frac{H_{s b}}{w_{s} T_{p}}\right) \cdot 10^{-5}
$$

Wave-energy flux

Considering a wave not normally incident to the shoreline, the wave energy flux is given by:

$$
F_{b}=E_{b} C_{g b} \cos \alpha_{b}
$$

where $E_{b}$ is the wave energy per unit crest, $C_{g b}$ is the group velocity and the subscript $b$ refers to the start of the breaking zone. For irregular waves, and using significant values at the breaker, the wave energy flux is:

$$
F_{b}=\frac{2^{\frac{5}{4}}}{8} \frac{g^{\frac{3}{2}}}{\sqrt{\gamma_{b}}} \rho H_{s b}^{\frac{5}{2}} \cos \alpha_{b}
$$

Energy dissipation is considered negligible before the breaker (bottom friction), so $F=F_{b}$ can be assumed. 
Mean (or representative) longshore current

The ideal scenario is when longshore current data is available. When it's not the case, $V$ can be calculated from wave characteristics and beach profile. Bayram et al. (2007) uses a simple longshore momentum equation which assumes linearized friction and neglects lateral mixing (Larson and Kraus, 1991):

$$
\frac{2}{\pi} \rho c_{f} u_{0} V=\frac{d S_{x y}}{d x}
$$

where $c_{f}$ is the friction coefficient, $u_{0}$ is the bottom orbital velocity and $S_{x y}$ is the radiation stress directed along the shore, and transported onshore. Assuming that shallow water conditions hold and that the beach profile can be approximately represented by a Dean's equilibrium beach profile $\left(h=A x^{2 / 3}\right.$, being $A$ the shape parameter (Dean and Dalrymple, 2004)), an expression for the longshore current can be written:

$$
V=\frac{5}{24} \frac{\pi \gamma_{b} \sqrt{g}}{c_{f}} A^{2} \frac{x^{\frac{1}{3}}}{\sqrt{h_{b}}} \sin \alpha_{b}
$$

The shape parameter $A$ can be related to the fall velocity $w_{s}$ with Eq.(9).

$$
A=\frac{9}{4}\left(\frac{w_{s}^{2}}{g}\right)^{\frac{1}{3}}
$$

Averaging $V$ in Eq.(8)along a cross-shore section ( $x$ direction):

$$
\bar{V}=\frac{1}{x_{b}} \int_{0}^{x_{b}} V d x=\frac{5}{32} \frac{\pi \gamma_{b} \sqrt{g}}{c_{f}} A^{\frac{3}{2}} \sin \alpha_{b}
$$

This formula for the longshore current, neglects the influence of lateral mixing. In equation Eq.(10) the friction coefficient is characterized in a simplistic manner (it is considered constant and equal to $0.005)$ and the wave climate is represented by a single representative wave. It should be noted that Eq. (10) is not directly dependent on wave height.

\section{Differences and similarities to other bulk formulas}

The Bayram formula and the CERC formula (CERC, 1984) share the basic premise that LST is directly correlated to the longshore component of wave-energy flux. Bayram et al. (2007) even calculated an expression for the transport coefficient $\varepsilon$ that makes the Bayram formula equivalent to the CERC formula. However, the Bayram formula only uses the wave energy flux to account for the sediment stirring, while the CERC formula doesn't distinguish between sediment stirring and longshore current generation.

The Kamphuis formula (Kamphuis, 1991) is almost entirely empirically derived and is therefore very different from the bulk formulas mentioned above.

\section{PERFORMANCE MEASURES ADOPTED IN THIS STUDY}

To evaluate the overall performance the formula, root mean square error $(R M S E)$ and bias were used. These values were calculated as:

$$
\begin{aligned}
R M S E & =\sqrt{\frac{\sum_{i=1}^{n}\left(\log \left(Q_{p, i}\right)-\log \left(Q_{m, i}\right)\right)^{2}}{n}} \\
\text { bias } & =\frac{\sum_{i=1}^{n}\left(\log \left(Q_{p, i}\right)-\log \left(Q_{m, i}\right)\right)}{n}
\end{aligned}
$$

The RMSE value is a commonly used error measure. The sum of squares gives more weight to higher error values, and consequently higher error variances. The bias value gives an indication of any systematical offset of the results. Because logarithmic values (base 10) are considered in all these statistical measures, the values indicate errors in terms of magnitude order, e.g., a RMSE value of one would mean that the predicted values can be roughly 10 times higher or smaller than the measured ones. Another measure of the performance used in this study, is the percentage of calculated values that are within a factor of 2 or 4 with respect to measured values. 


\section{IMPROVING THE FORMULA}

\section{Dependency of the calibration factor on physical parameters}

The Bayram formula is based on a simplified model that attempts to simulate the basic physical processes. The calibration coefficient is expected to take into account effects that are not included in the basic models (or that are poorly represented).

The formula can be written in a simplified manner:

$$
Q=\varepsilon X
$$

where $Q$ is the LST rate, $\varepsilon$ is the calibration coefficient (also designated as the transport coefficient) and $X$ is a function of the input data.

For each data point $i$ a comparison was made in terms of the differences between the logarithms (base 10) of $X$ and measured values: $\Delta i=\log (i)-\log (Q m, i)$. The $\Delta i$ values will be henceforth called deltas.

With the intent of finding the optimal $\varepsilon$ coefficient, it is necessary to find a dependency of the deltas with some physical parameter. Being $\varepsilon$ non-dimensional by definition, the dependency will be with one or more than one non-dimensional parameters, or with a non-dimensional combination of dimensional parameters. The parameters were chosen considering the dimensional analysis made in Bayram et al. (2007) and Kamphuis (1991), adding the surf similarity (related to the breaker type). The chosen parameters were: $H_{s b} L_{o}, H_{s b} / D_{50}$, surf similarity $\mathrm{m} / \sqrt{H_{b} / L_{0}}$ and the Dean number: $H_{s b} / w_{s} T_{p}$.

In order to find a correlation and study the data distribution, the deltas were plotted against the above mentioned non-dimensional parameters. The plots show considerable scatter (Figure 3). To help identifying a trend, it was important to study the (y-axis) distribution of the deltas. Considering that the data set has a sufficient number of data points, collected with different methods, it is reasonable to assume that the measurement errors are normally distributed and centered on zero. A normal distribution of the deltas will only be visible if the error due to the formulation itself is not much higher than the measurement error. For this reason, it is hypothesized that the normality of the $y$-axis deltas distributions is a measure of the adequateness of the calibration coefficient because it means that measurement error is prevailing. The evolution of these distributions along the $x$-axis can then be a precious help to choose the best non-dimensional parameter for the calibration coefficient.

In order to have enough points to calculate a statistically meaningful distribution across the $y$-axis, a moving window method was used. Basically this method consists in calculating a number of histograms from sets of 60 points, each one beginning 5 points further along the sorted $x$ values. The method can be illustrated in Figure 2 that shows a scatter plot of the deltas (calculated with the CERC formula) vs. $H_{s b} L_{o}$. In this figure the distance between histograms is exaggerated for visualization purposes.

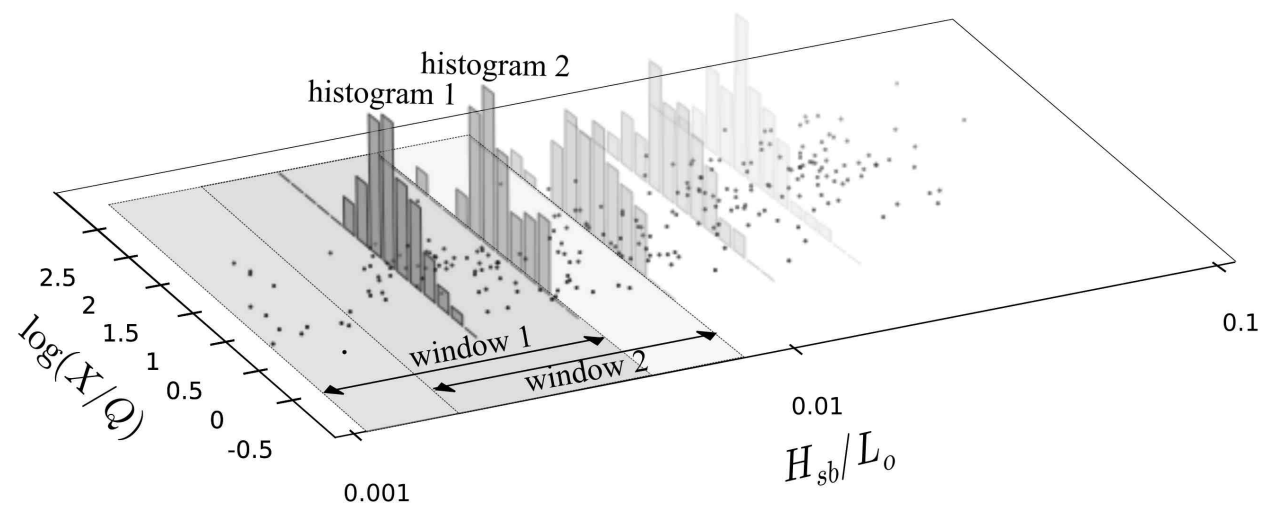

Figure 2 - Moving window method to estimate the evolution of the distribution along the x-axis. Histogram 1 refers to the points contained on window 1 and Histogram 2 to window 2.

Having a significant number of histograms, the evolution along the $x$-axis of the distributions can be plotted as a contour. Figure 3 shows the contours of the distributions for the non-dimensional parameters considered: the Dean number, surf similarity, $H_{s b} L_{o}$ and $H_{s b} b D_{50}$. In the same figure it can be 
observed that the range covered by the contours is different for the non-dimensional parameters considered. This is caused by the different distribution of points along the $x$-axis, being $H_{s b} L_{o}$ the parameter where the points are the most uniformly distributed. In this image one can also notice that the distribution with the $H_{s b} L_{o}$ parameter follows a more or less clear trend and that for the others parameters the existence of a trend is less clear.

It is important to study the normality of the distributions, i.e. the probability that the sample of $n$ points considered for each histogram came from a normal distribution. To this effect, an AndersenDarling (Andersen and Darling (1952)) test was used. In this test, a statistic $A^{2}$ is calculated. If this statistic is above the critical value for a given significance level, then the null hypothesis (the sample is taken form a population normally distributed) can be rejected. The chosen value for the significance level was $5 \%$. The critical value for that significance level is 0.74 (Figure 4). The mean values of $A^{2}$ were: 1.20 for the Dean number, 1.19 for the surf similarity, 0.46 for $H_{s b} L_{o}$ and 0.83 for $H_{s b} D_{50}$. This leads to the conclusion that the distribution of deltas, when expressed as a function of the $H_{s b} L_{o}$ parameter, is clearly the most likely to have been taken from a normal distribution.

For the two reasons explained above (distribution along $x$-axis and normality), the best expression to describe the deltas is a function of the $H_{s b} L_{o}$ parameter for the Bayram formula.
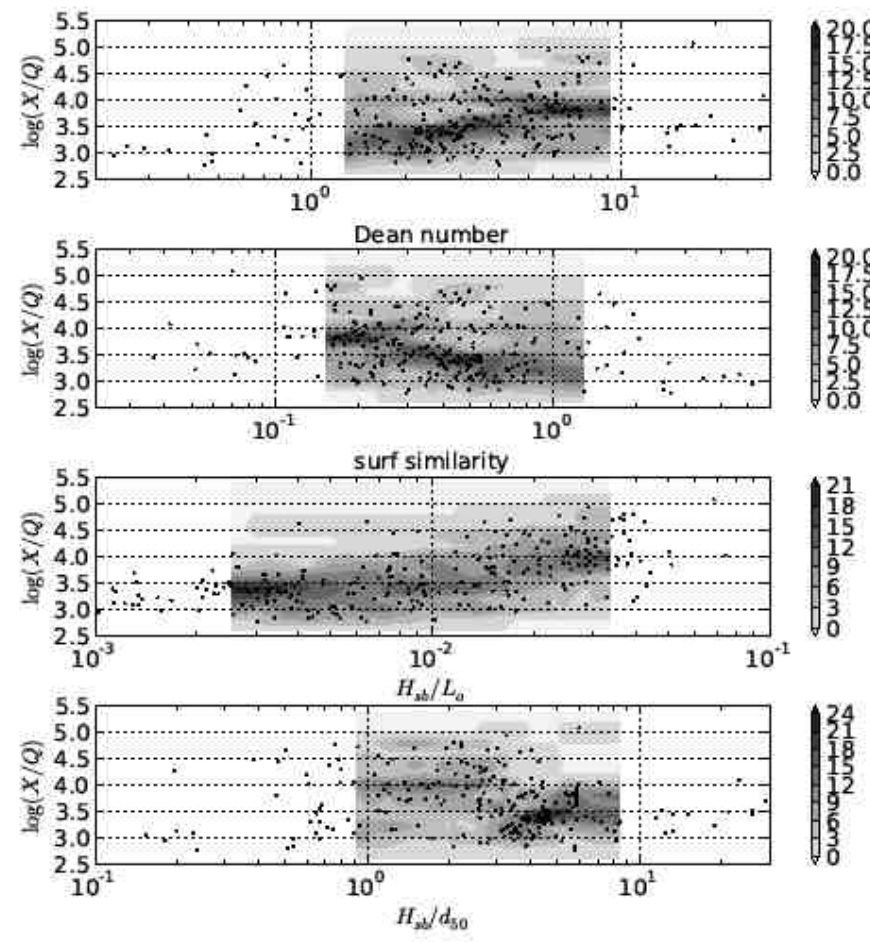

Figure 3 - Distribution of deltas for the non-dimensional parameters considered: Dean number, surf similarity, $H_{s b} L_{o}$ and $H_{s b} D_{50}$ (using the Bayram formula). 

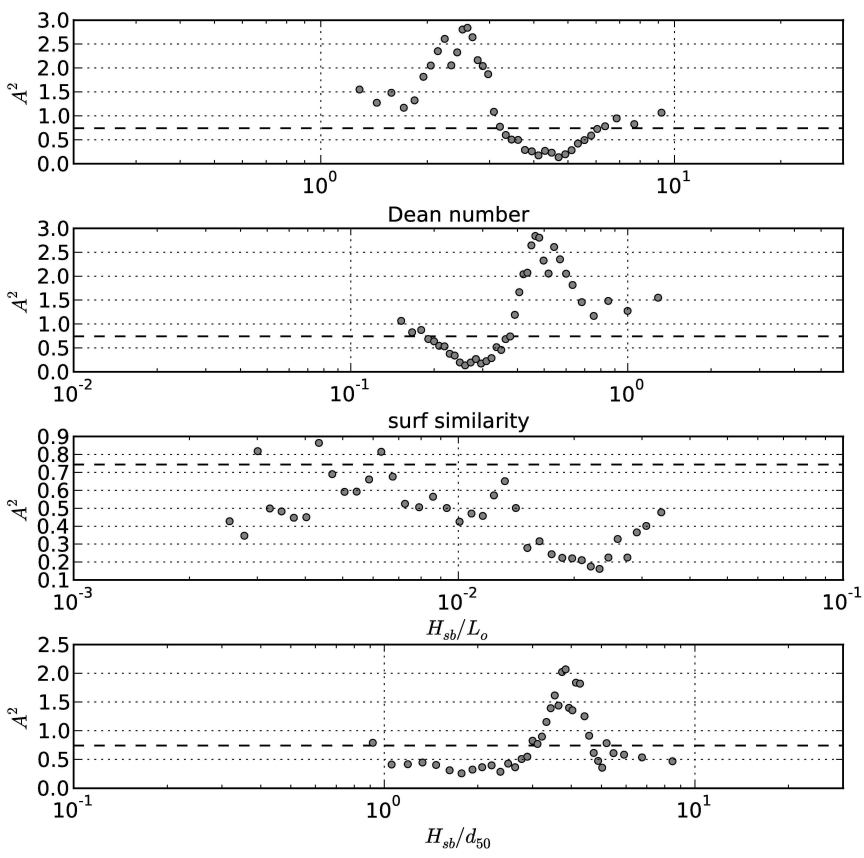

Figure 4 - Anderson-Darling statistic for the dimensionless parameters considered: Dean number, surf similarity, $H_{s b} L_{o}$ and $H_{s b} D_{50}$ (using the Bayram formula).

\section{Least-squares optimization}

All optimization calculations were carried out using a modified Levenberg-Marquardt (Levenberg, 1944) least-squares optimization method. The method takes a vector of data points $y_{i}$ and tries to find the set of parameters $x$ for the function $g(t, x)$ such that the squared sum of residuals becomes minimal. The residuals are defined as $f_{i}(x)=y_{i}-g\left(t_{i}, x\right)$, where $t$ is the available input data. The LevenbergMarquardt algorithm is an iterative method. It starts with an initial guess $x_{n=} x_{0}$, and in each iteration the algorithm determines a correction $p$ to $x_{n}$ that produces a sufficient decrease in the residuals calculated with the new parameter set $x_{n+1}=x_{n}+p$. The solution will converge to $x^{*}$, being $g\left(t, x^{*}\right)$ the function minimizes the squared sum of residuals. The calculation of the correction $p$ is described in detail in Moré et al. (1980).

This method allows for nonlinear models to be tested. However, depending on the initial guess, the Levenberg-Marquardt algorithm can converge to local minima, failing to find the absolute minimum.

Taking into account the apparent trend in Figure 3, two non-linear functions were considered: one polynomial and the other exponential. The expressions for the deltas take the form: $\Delta_{i}(x)=\log [f(x)]$ where $f(x)=a x^{b}+c$ (polynomial function) or $f(x)=a e^{b x}+c$ (exponential function) and $a, b$ and $c$ are the coefficients to be calculated. The calibration coefficient becomes: $\varepsilon=[f(x)]^{-1}$, as can be seen relating Eq.(13) with: $\log \left(\frac{X}{Q}\right)=\log [f(x)]$ :

The optimization was carried out for the non-dimensional $H_{s b} L_{o}$ parameter considered in the previous section. The best result was:

$$
\varepsilon_{n e w}=\left(7.862 \times 10^{5}{\frac{H_{s b}}{L_{o}}}^{1.283}+1672.2\right)^{-1}
$$

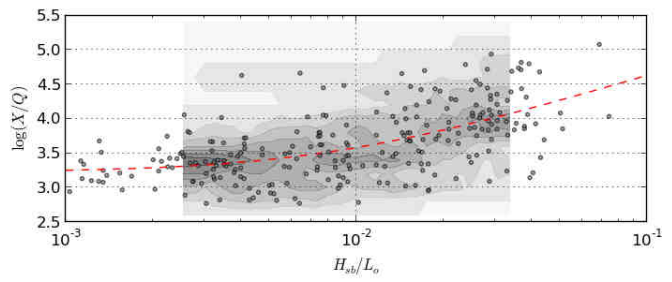

Figure 5 - Best fitting function (dashed line), point scatter and distribution contour for the Bayram formula 
The exponential function resulted in a slightly higher RMSE (0.4074 against 0.4069 of the polynomial).

\section{RESULTS}

Figure 6 and Figure 7 show the $Q_{\text {predicted }} v s$. $Q_{\text {measured }}$ plots with the original and new coefficients under the data set used in this study. With the new coefficient, there is still considerable scatter, which is to be expected due to the very complex nature of the processes involved, and the difficulty of measuring all parameters related to LST. These simplified models fail to take into account factors such as the existence of bars and other morphological features on the beach that can influence the current patterns or the wave breaking. The existence of a bar may drastically influence the value of the beach slope at the breaker zone, when compared with the values calculated with a representative Dean profile (as assumed in the Bayram formula). Probably there is also some noise in the data due to currents generated by forcing mechanisms other than wave breaking (e.g. wind and tides). Another source of uncertainty is the use of representative wave conditions to synthesize the wave climate during a period of time. This is a problem mainly in long term measurements.

It is possible to use the Bayram formula with measured current data or with different formulations for the mean longshore current, accounting for other forcing mechanisms. However, in this study, the mean longshore current was always calculated from wave conditions, and measured current data was not used.

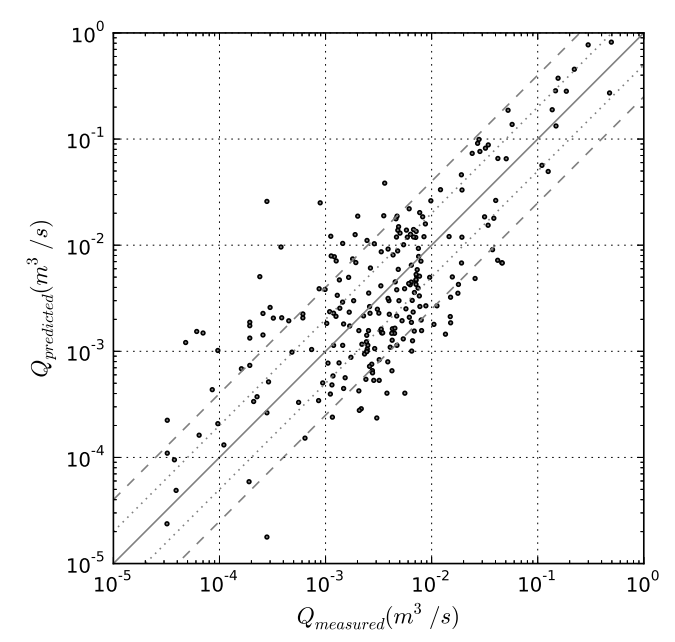

Figure $6-Q_{\text {predicted }}$ vs. $Q_{\text {measured }}$ using the Bayram formula with the original coefficient. Solid line corresponds to $x=y$, dotted lines to $x=0.5 y$ and $x=2 y$ (factor of 2) and dashed lines to $x=0.25 y$ and $x=4 y$ (factor of 4) 


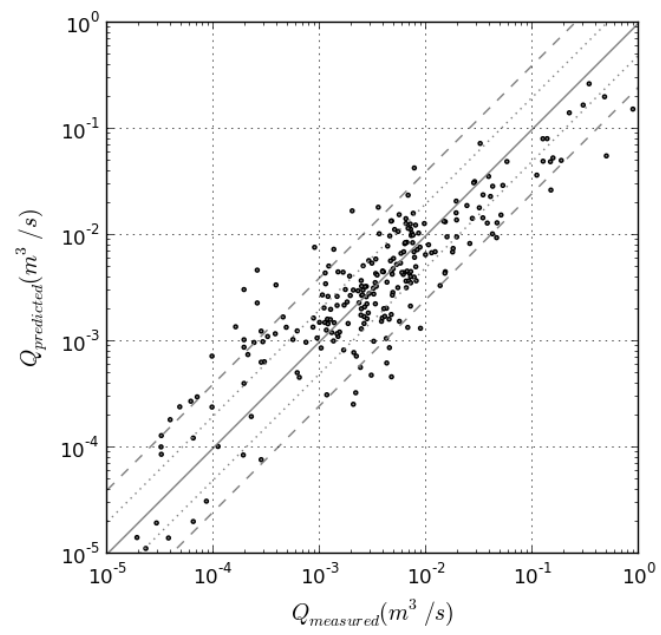

Figure 7 - $Q_{\text {predicted }}$ Vs. $Q_{\text {measured }}$ using the Bayram formula with the new coefficient. Solid line corresponds to $x=y$, dotted lines to $x=0.5 y$ and $x=2 y$ (factor of 2) and dashed lines to $x=0.25 y$ and $x=4 y$ (factor of 4)

The performances of the original and the new coefficients are compared in Table 1.

\begin{tabular}{|c|c|c|c|c|}
\hline & $R M S E$ & bias & Factor of 2 & Factor of 4 \\
\hline Original coefficient & 0.570 & 0.01 & $32 \%$ & $71 \%$ \\
\hline New coefficient & 0.407 & 0 & $56 \%$ & $85 \%$ \\
\hline
\end{tabular}

Table 1 shows that a significant improvement was achieved with the new coefficient, both in RMSE values and the percentage of points between factors of 2 and 4 with respect to measurements. The bias value is indistinguishable from zero.

The breaking wave height to deep water wavelength ratio $H_{s b} L_{o}$ (which is similar to the wave steepness), present in the new coefficient may affect LST in more than one way. Smaller wave steepness is usually the result of a large $T_{p}$, which can have opposite effects on the LST. A larger period gives more time for the sediment to settle between waves and yields smaller wave breaking angles, due to more intense refraction. Both these effects would result in a reduction of the LST relatively to shorter period waves. On the other hand, a larger wave period also corresponds to a higher surf similarity parameter which is known to be associated with more intense plunging breakers (Battjes, 1974). This type of breakers dissipates energy in a concentrated area, stirring more sediment from the bottom, thus resulting in higher LST. The present analysis suggests that the latter effect is more important than the former, as LST increases with $T_{p}$ in Eq.(14).

\section{Model generality}

Two methods were used to access the generality of the models presented in the previous section: cross-validation and bootstrapping.

The cross-validation method is one way to infer the generality of the model. The data set is randomly divided in two groups: the calibration group and the validation group. In this case each group had $50 \%$ of the data points. The calibration group will be used to calculate the coefficients, using the least-squares algorithm described in a previous section. The validation group will be used to test the predicting skill of the model. Because there is a random element, with each division of the data set different coefficients and different statistics will be obtained. In order to evaluate the variability of the results, the procedure was repeated 10000 times and the distributions of coefficients and statistics were determined. 
Another way to investigate the generality of the model and the confidence level of the results, is the bootstrapping method. In this method, the coefficients and statistics of a high number of samples taken from the available data set are calculated. In basic terms, the method can be summarized by the following steps: first, a sample with random points (picked with replacement, i.e. the same number can be picked more than one time) is selected. This so called bootstrap sample has the same size as the data set. A least-squares fitting is performed using the bootstrap sample, and the coefficients and statistics are stored. The process is repeated many times (in this case 10000). Using the stored values of all the fitting operations, it is possible to calculate distributions for the estimated parameters and statistics, and evaluate their uncertainty.

Each bootstrap sample has elements that are repeated and therefore there are points of the data set that are not present in the sample. This means that the coefficients obtained by the fitting process using the bootstrap samples were derived with less data points. The resulting distributions show the variability of the model when its parameters are derived with less data (as in the cross-validation method).

Both cross-validation and bootstrapping methods were used to verify the generality of the Bayram formula with the new coefficient, and the statistics of the RMSE and bias distributions were determined. The median, standard deviation and $95^{\text {th }}$ percentile of the RMSE distributions are shown on Table 2. The statistics obtained for the bias distribution are all indistinguishable from zero. For that reason they are not shown in the table.

\begin{tabular}{|c|c|c|c|}
\hline & median & Standard deviation & $95^{\text {th }}$ percentile \\
\hline Cross-validation & 0.414 & 0.0199 & 0.446 \\
\hline bootstrapping & 0.404 & 0.0192 & 0.436 \\
\hline
\end{tabular}

The resulting histograms for the RMSE values are shown in Figures 8 and 9. The results of the two methods are almost identical. In Table 2 it can be seen that the RMSE values are in the vicinity of 0.41 , and have a small standard deviation (circa 0.02). The $95^{\text {th }}$ percentile is equal or less than 0.45 , in all formulations. This means that more than $95 \%$ of the samples have an RMSE value well under the one obtained with the previous coefficient (0.57). The low RMSE variability indicates that the improved coefficients have good generality.

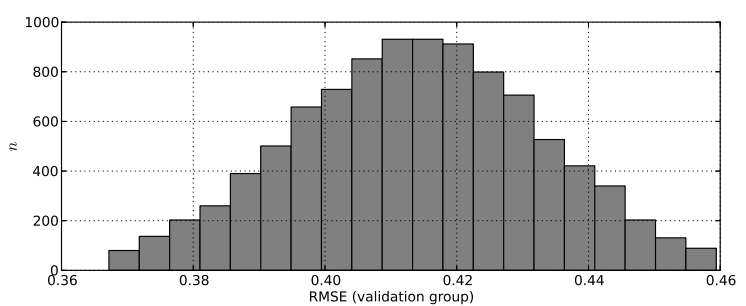

Figure 8 - Histograms of RMSE values, using the calibration-validation method.

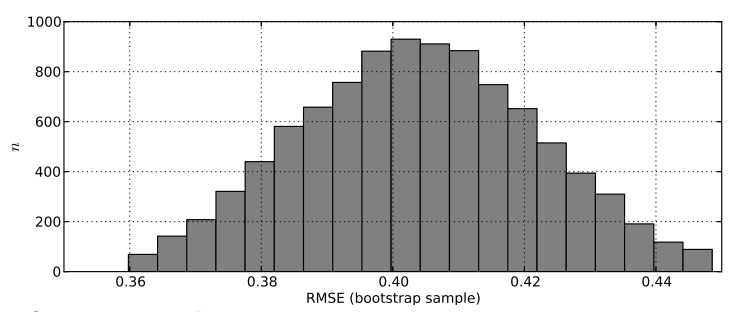

Figure 9 - Histograms of RMSE values, using the bootstraping method.

\section{CONCLUSIONS}

A comprehensive analysis of the bulk LST formula presented in Bayram et al. (2007) has been undertaken. The analysis resulted in new calibration coefficient, taking advantage of a least-squares optimization algorithm that allows the use of non-linear functions. The predictive skill of the Bayram 
formula $(R M S E=0.407$, bias $=0$, \% within factor $2=56 \%$ ) was significantly better than the previous version $(R M S E=0.57$, bias $=0.01$, \% within factor $2=32 \%$ ). The generality of the improved formulas was examined by applying the bootstrapping and cross-validation statistical methods, both of which returned similar results and confirmed the generality of the formulations. It is important to notice that, despite the significant improvement in the prediction skills of the Bayram formulation, there is still considerable scatter and $44 \%$ of the predictions deviate more than a factor 2 with respect to observations. This may be due to the several reasons including: the non-consideration of parameters that may influence LST such as cross-shore profile features, 3 dimensional morphological features, tidal range and wind conditions in the Bayram formula. Other sources of error may be the experimental errors and the fact that there is insufficient data for high LST conditions. However, the study of the deltas distributions was only meaningful because experimental errors were present and something about their nature was assumed. This assumption, that given the high number of data points, the measurement error is expected to be normally distributed, centered on zero and that a more adequate coefficient would yield more normal y-axis distribution (assessed using the Anderson-Darling statistic criterion), resulted in a significant improvement of the calibration coefficient. This can be considered as an indication that the assumption was reasonable.

\section{ACKNOWLEDGEMENTS}

The authors wish to express their gratitude to Dr. Atilla Bayram (Halcrow, Inc.) and Prof. Magnus Larson (Lund University, Sweden) for kindly providing the invaluable data set used herein. This study was funded by "Fundação para a Ciência e a Tecnologia" via the First author's PhD program. The authors also acknowledge the contribution of Peter van Gelder for his help and insights in the statistical analysis.

\section{REFERENCES}

Anderson, T. W., and D.A. Darling. "Asymptotic Theory of Certain 'Goodness of Fit' Criteria Based on Stochastic Processes.” The Annals of Mathematical Statistics 23, no. 2 (June 1952): 193-212.

Bailard, James A. "An Energetics Total Load Sediment Transport Model For a Plane Sloping Beach." Journal of Geophysical Research 86, no. C11 (1981): 954.

Battjes, J.A. "Surf Similarity." Proceedings of the 14th International Conference on Coastal Engineering 1 (1974): 466-480.

Bayram, Atilla, Magnus Larson, and Hans Hanson. "A New Formula for the Total Longshore Sediment Transport Rate." Coastal Engineering 54, no. 9 (2007): 700-710.

Bijker, E. W. "Littoral Drift as a Function of Waves and Current." In Proc. 11th Conf. Coastal Eng., 1:415-435. London, 1968.

CERC. Shore Protection Manual. Vol. I and II. Vicksburg: Coastal Engineering Research Center, USACE, 1984.

Dean, Robert G., and Robert A. Dalrymple. Coastal Processes with Engineering Applications. Cambridge University Press, 2004.

Deigaard, R., J. Fredsoe, and IB Hedegaard. "Mathematical-model for Littoral Drift.” Journal of Waterway Port Coastal and Ocean Engineering -ASCE 112, no. 3 (May 1986): 351-369.

Hanson, H. "GENESIS - a Generalized Shoreline Change Numerical Model.” Journal of Coastal Research 5, no. 1 (1989): 1-27.

Kamphuis, J. William. “Alongshore Sediment Transport Rate.” Journal of Waterway, Port, Coastal and Ocean Engineering 117, no. 6 (1991): 624.

Larson, Magnus, and Nicholas C. Kraus. "Numerical Model of Longshore Current for Bar and Trough Beaches.” Journal of Waterway, Port, Coastal, and Ocean Engineering 117, no. 4 (July 1991): 326-347.

Levenberg, K. "A Method for the Solution of Certain Non-linear Problems in Least Squares." Quarterly Journal of Applied Mathmatics II, no. 2 (1944): 164-168.

Miller, Herman C. "Comparison of Storm Longshore Transport Rates to Predictions". Copenhagen, Denmark, 1998.

Moré, JJ, BS Garbow, and KE Hillstrom. User Guide for MINPACK-1. Argonne National Laboratory, 1980. http://www.mcs.anl.gov/ more/ANL8074a.pdf. 
Sanil Kumar, V., N. M. Anand, P. Chandramohan, and G. N. Naik. "Longshore Sediment Transport Rate--measurement and Estimation, Central West Coast of India." Coastal Engineering 48, no. 2 (April 2003): 95-109.

Schoonees, J.S., and A.K. Theron. "Review of the Field-data Base for Longshore Sediment Transport." Coastal Engineering 19, no. 1-2 (1993): 1-25.

Smith, E. R, P. Wang, and J. Zhang. "Evaluation of the CERC Formula Using Large-scale Model Data." In Proceedings of Coastal Sediments. Vol. 3, 2003.

Wang, P., N. C. Kraus, and R. A. Davis. "Total Longshore Sediment Transport Rate in the Surf Zone: Field Measurements and Empirical Predictions." Journal of Coastal Research 14, no. 1 (WIN 1998): 269-282.

WL|Delft Hydraulics. UNIBEST, A Software Suite for Simulation of Sediment Transport Processes and Related Morphodynamics of Beach Profiles and Coastline Evolution. Model Description and Validation. Delft hydraulics Report, 1992. 